\title{
Influenza vaccination in the elderly
}

\author{
Rosemary Morgan, Debra King
}

\section{Summary}

There is overwhelming evidence for the clinical efficacy of the influenza vaccine, especially in the elderly with chronic disease, reducing mortality and hospital admissions. There is also evidence to suggest that the influenza vaccine may be beneficial in the healthy elderly. There is some evidence to suggest that the antibody response in the elderly to the vaccine may decrease with increasing age, although there are several confounding factors that have not been taken into account in many of these studies. That aside, even if antibody response is not as good as that in younger people, the evidence that vaccination saves lives and reduces morbidity in the elderly means that the vaccination should be offered to elderly patients at high risk and perhaps even to the elderly healthy population. Although vaccination of an elderly at-risk patient does not necessarily mean that that particular patient will mount an appropriate antibody response, a significant number of elderly patients will respond appropriately. Serious side-effects from vaccination are extremely rare and the more common side-effects are mild and self-limiting. Increasing the number of elderly people receiving the influenza vaccination will not only result in cost savings for the National Health Service in terms of reduced hospitalisation but, more significantly, the elderly will benefit in terms of reduced morbidity and mortality.

Keywords: influenza vaccination, elderly

Geriatric Medicine, Withington Hospital, Nell Lane, West Didsbury, Manchester M20 8LE, UK

R Morgan

Arrowe Park Hospital, Arrowe Park Road, Wirral, Merseyside

L49 5PE, UK

D King

Accepted 3 October 1995
In the UK influenza epidemics are an important cause of preventable mortality and morbidity, especially in the elderly. The 1989-90 epidemic killed an estimated 26000 people over a 12-week period, most of whom were elderly with chronic medical conditions. ${ }^{1,2}$

Although influenza vaccines are poor at preventing infection, they reduce the incidence of bronchopneumonia, hospital admissions and mortality in those most at risk. ${ }^{3}$ The Department of Health (DOH) recommends the influenza vaccine to particular high-risk groups on an annual basis (box 1), while in the USA old age per se ( $>65$ years of age) is an indication for vaccination.

Despite these recommendations several studies have shown that $50 \%$ of those at high risk do not receive the vaccine in the UK. ${ }^{4,5}$ Consequently deaths and hospitalisations occur which could have been prevented. Concerns include possible side-effects of vaccination, as well as the level of seroconversion in old age. This leads to a lack of conviction of the physician. Together with an absence of co-ordination in targeting the at-risk population, this results in a substantial shortfall in patient vaccination.

\section{The influenza virus}

There are three influenza viruses, A, B and C, which differ in their internal nucleoproteins. The surface of the influenza virus is coated with spikes of haemagglutinin and an enzyme, neuramidase, which enables the virus to attach itself to the respiratory epithelium of the host and consequently cause infection to susceptible cells.

Pandemics, which occur approximately every 10 years, are caused by 'antigenic shift' of influenza A resulting in the appearance of an influenza virus with a new haemagglutinin ( $\mathrm{H}$ antigen) or neuramidase $(\mathrm{N}$ antigen) subtype. Influenza A viruses mainly affect man but may also cause infection in a wide range of mammals and birds; new strains might emerge during passage between mammals, birds and human populations. During interpandemic periods antigenic variability is less marked and is caused by 'antigenic drift'. This involves minor antigenic changes resulting from the accumulation of random point mutations which result in alterations in the amino acid composition of haemagglutinin and neuramidase. This slow and more gradual change does not necessarily occur each year. Epidemics arise if new strains of influenza A and B are significantly different from previous strains encountered by the population. Emergence of a new strain is followed by an increase in prevalence to a peak in two or three years, so that at any time there are two strains of influenza $A$ and one of B which dominate the epidemic scene (box 2). The elderly are more susceptible and vulnerable to many infections than younger adults and there is a five-fold excess death rate among persons aged 65 years or more from influenza compared to younger adults. ${ }^{6}$ In part, this is due to a decline in the immune response that occurs with ageing, in particular the decline in cell-mediated immunity. Thymic involution leads to the appearance of anti-self-reactive $T$ lymphocytes and $\mathrm{T}$ lymphocytes that do not express self-MHC restricted antigen recognition. ${ }^{7}$ There are also changes in the ratio of T-cell subsets, eg, the percentage of CD4+ increases with increasing age whereas CD8+ declines. ${ }^{8}$ In addition, the production of some cytokines, eg, interleukin II, declines with age. ${ }^{9}$

The clinical consequences of a decline in cell-mediated immunity include reactivation of latent fungal, myobacterial and viral disease. Although the function of the B lymphocyte appears not to change with age, the optimal production of antibodies may be affected with age since the humoral response to antigen requires processing via $\mathrm{T}$ lymphocytes.

\section{Complications of influenza}

Influenza may produce several complications, some of which are more common in the elderly (box 3 ). The single most important complication is pneumonia. 


\begin{tabular}{|l|}
\hline Influenza vaccination: \\
indications (DOH) \\
\hline - chronic respiratory disease \\
- chronic heart disease \\
- chronic renal disease \\
endotes mellitus and other \\
- immunosuppressed patients \\
- residents of nursing homes and \\
other long-stay facilities \\
\hline
\end{tabular}

Box 1

\begin{tabular}{|lll|}
\hline \multicolumn{3}{|l|}{$\begin{array}{l}\text { Examples of pandemic } \\
\text { influenza strains }\end{array}$} \\
\hline $\begin{array}{l}\text { Time pre- } \\
\text { valence }\end{array}$ & $\begin{array}{l}\text { Colloquial } \\
\text { name }\end{array}$ & $\begin{array}{l}\text { Antigenic } \\
\text { description }\end{array}$ \\
1947 & A1 & HIN1 \\
$1957-68$ & Asian & H2N2 \\
1968 & $\begin{array}{l}\text { Hong } \\
\text { Kong } \\
\text { Russian }\end{array}$ & H3N2 \\
1977 & H1N1 \\
& & \\
\hline
\end{tabular}

Box 2

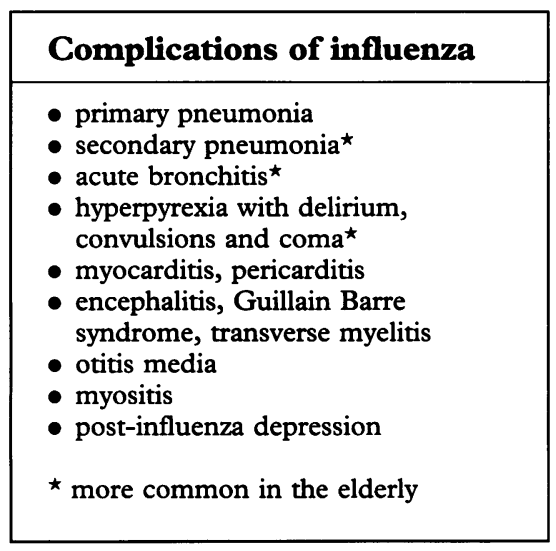

Box 3
Primary influenza pneumonia typically occurs approximately $36 \mathrm{~h}$ after onset of flu-like symptoms and may result in respiratory failure and death. It is more common in patients with prior cardiac or pulmonary disease.

Secondary bacterial pneumonias occur because of the ability of the influenza virus to impair mucocilliary function and to reduce the capacity of the phagocytic cells in the airways to clear bacteria away. ${ }^{10}$ Bacterial pneumonias typically occur three and five days after the onset of influenza. Streptococcus pneumoniae and Staphylococcus aureus are the most common bacterial pathogens.

In addition to changes in the immune system and the increased incidence of multiple pathology (including cardiac and pulmonary disease) with age, the elderly are more vulnerable to pneumonia because of changes in their respiratory systems. Age-related reduction in chest wall expansion and alveolar elasticity contribute to a dimished cough reflex in the elderly. ${ }^{11}$ There is also evidence of decreased function of the mucocilliary transport system in the lungs of elderly people. ${ }^{12}$

The risk of acquiring pneumonia may also increase according to the environment. Elderly patients (age 75 years and over) in nursing homes have been shown to have a two- to three-fold higher risk of developing pneumonia than elderly people in the community. ${ }^{13}$

\section{The clinical effectiveness of vaccination}

Several observational studies looking at the effectiveness of the influenza vaccination have been conducted in nursing home populations. In these more vulnerable patients, influenza vaccination reduces episodes of pneumonia by $50-60 \%$ and is $60-70 \%$ effective in preventing death. ${ }^{14,15}$

A randomised double-blind placebo-controlled trial, ${ }^{16}$ assessed the efficacy of the influenza vaccination of the elderly during the influenza season of 1991/2. High-risk groups were excluded. Over 1800 elderly people living in the community were randomised to receive either placebo or vaccine. All had blood assays for antibody titres pre- and post-vaccination and were asked to complete a questionnaire about their experience of flu-like illnesses. The incidence of serological influenza was halved in the vaccinated group. This would support the efficacy of the influenza vaccine in the healthy elderly as well as those with prior disease and would confirm the findings of three recent case-controlled studies that demonstrated the efficacy of the influenza vaccine in the noninstitutionalised elderly. ${ }^{17-19}$

In a recent large cohort study ${ }^{20}$ elderly people over the age of 65 years living in the community were assessed for the rate of vaccination and the occurence of influenza and its complications in each of three seasons, 1990/1, 1991/2 and 1992/3. Each cohort included more than 25000 people over the age of 65 years. Immunisation rates ranged from $45 \%$ to $58 \%$. Vaccination was associated with significantly fewer hospitalisations for pneumonia and influenza $(48-57 \%$ reduction, $\mathrm{p}<0.002)$. Vaccination was also associated with a $37 \%$ reduction $(p=0.04)$ in the rate of hospitalisation for congestive heart failure during the 1991/2 season, when influenza $A$ was epidemic. It was also associated with reductions of $39 \%$ to $54 \%(p=0.001)$ in mortality from all causes during the three influenza seasons.

\section{Cost effectiveness}

The cost of the influenza vaccine is substantially less than the costs of almost all other preventative and therapeutic interventions that are used in older people. ${ }^{21}$ Several studies, ${ }^{20,22}$ have demonstrated the cost effectiveness of the influenza vaccine. In a large cohort study, ${ }^{20}$ the costs of hospitalisation for all types of illness studied were lower in the vaccinated group. Direct savings per year averaged $\$ 117$ per person vaccinated (range $\$ 21$ to $\$ 235$ ) with cummulative savings of nearly $\$ 5$ million. This figure is higher than other estimates of savings produced by the influenza vaccine. This study, however, took into account reductions in costs of hospitalisation for congestive heart failure and acute and chronic respiratory conditions other than pneumonia and influenza.

\section{Influenza vaccine-seroconversion}

Protection against influenza is generally due to adequate titres of antibody against the haemagglutinin antigen, ie, serum HAI antibodies titres of $1: 40$ or greater. One of the major concerns regarding influenza vaccination is that, with increasing age, antihaemagglutinin antibody response to influenza vaccine may decline. The proportion of elderly who develop serum HAI antibody titres of $>1: 40$ post-vaccination ranges from more than $85 \%$ for $\mathrm{H} 3 \mathrm{~N} 2$ vaccine 
antigens for healthy elderly people in the community to $46-100 \%$ for $\mathrm{H} 3 \mathrm{~N} 2$ vaccine antigen and $20-69 \%$ for $\mathrm{B}$ antigens for ambulatory elderly in nursing homes. ${ }^{23}$ However, review of comparative studies between young and old and clinical correlation between serum antibody levels and clinical protection are often confounded by failure to account for previous vaccination, small sample sizes and increased likelihood of Type II errors, exlusion of immunocompromised subjects, and high titres of prevaccination antibodies. ${ }^{24}$

Although some studies have reported that elderly people with chronic disease respond less well to influenza vaccination than healthy subjects, other studies which have excluded immunocompromised people have found that other chronic illnesses do not appear to have a major effect in antibody response to influenza vaccine. ${ }^{25}$

\section{The influenza vaccine}

Inactive influenza virus vaccine have been the principle means of preventing influenza since the late 1940s. Recent vaccines have consisted of highly purified egg-grown inactivated viruses in a trivalent preparation containing two type A strains and one type B strain. Since the vaccine contains only non-infected viruses it cannot cause influenza.

The potential use of live attenuated influenza vaccines has been of interest in younger subjects. Immunity conferred by killed (inactivated) vaccines is often inferior to that resulting from infection with live organisms. In part this is because replication of the living microbe confronts the host with a larger and more sustained dose of antigen. In addition, the immune response takes place largely at the site of natural infection, (eg, respiratory mucosa in influenza). Injected inactivated preparations of the virus may stimulate antibody synthesis in the spleen and lymph nodes without initiating an adequate immune response in local lymphoid tissue. However, in elderly people local IgA and systemic IgG anti-haemagglutinin responses are lower and shorter lasting following immunisation with live attenuated vaccine compared to inactivated vaccine. ${ }^{26}$ It is likely that age-related changes in the immune system result in a diminished immune response at the site of natural infection with a poor local antibody response. Thus, in the elderly, vaccination with live virus does not appear to be a suitable alternative to inactivated vaccines.

\section{Side-effects of the influenza vaccine}

\begin{tabular}{|l}
\hline Influenza vaccine \\
\hline Side-effects \\
- local erythema \\
- mild systemic illness \\
- demyelination syndromes (rare) \\
Contraindications \\
- allergy to eggs \\
- previous adverse reaction
\end{tabular}

Box 4

The commonest side-effect is an acute local reaction to the intramuscular injection. This occurs in approximately a third of vaccinations and lasts one to two days. Occasionally (less than $1 \%$ ) a mild systemic reaction with low-grade fevers occur with or without a flu-like illness, which can persist for one to two days.

In a randomised double-blind placebo-controlled study, ${ }^{27}$ involving over 1800 patients over the age of 60 years, soreness at the injection site was the only symptom that occurred more frequently following vaccination compared to placebo.

Rarely, post-demyelination syndromes have been reported following vaccination. There are no studies at present to suggest an increased risk of reaction in persons with multiple sclerosis or other demyelinating diseases. Contraindications to the influenza vaccine include a severe reaction to a previous dose or a history of anaphylactic hypersensitivity to eggs or egg protein.

\section{Alternatives to influenza vaccination}

Amantadine has been shown to be an effective prophylactic and therapeutic agent against influenza $A .{ }^{28}$ It leads to a reduction in viral shedding and shortens the duration of symptoms if started within $48 \mathrm{~h}$ of onset of influenza. The Department of Health recommends amantadine for previously nonimmunised at-risk patients for two weeks while the vaccine takes effect during an epidemic. It is also useful if an epidemic new strain occurs which has not been incorporated into the previously administered vaccine. Amantadine should also be considered during an influenza epidemic for elderly at-risk patients for whom the influenza vaccine is contraindicated. Side-effects of amantadine include insomnia, headaches and dizziness. Rimantadine has been shown to be an effective treatment for influenza and may be less toxic than amantadine. ${ }^{29}$ Immunisation is a more reliable form of prophylaxis since it does not require the patient to take medications. 


\section{Strategies for increasing uptake of influenza vaccination}

- identification of high-risk patients (data base)

- reminders in hospital discharge letters if high risk and not vaccinated

- health education

- computer-generated reminders

- delegation of responsibility for vaccination to the practice/district nurse

Box 5

\section{Strategies for increasing uptake of the vaccine}

Despite current recommendations on who should received the influenza vaccine, several studies have shown that more than $50 \%$ of those at high risk do not receive it. ${ }^{4,5}$ For patients to be successfully immunised they have to be willing to receive the vaccine from their doctor. Several studies have looked at the relationships between knowledge, attitudes and vaccination behaviour in patients. The major deterrent to vaccination seems to be concern about sideeffects. ${ }^{4}$ If physicians recommend the influenza vaccine to their patients, a high proportion will accept vaccination regardless of their initial attitude. ${ }^{30}$ The key to effective vaccination is to make sure that all patients who should be immunised are actually offered vaccination.

\section{Is vaccination appropriate for everyone?}

There are some elderly people who are severely disabled with little quality of life for whom vaccination may be inappropriate. It is important to discuss the implications of selectively withholding vaccination with individual patients and also with their relatives if appropriate. Obviously in the case of a mentally competent patient they should be asked permission if it is thought that their relatives/carers should be involved in the discussion. However, it must be remembered that such 'selective non-vaccination policies' might disadvantage other fitter elderly patients who share their facilities who might then be infected because of reduced 'herd' immunity. This produces an ethical dilemma for the doctor but as consent is required for influenza vaccination the full implications of refusal both for the individual patient and his/her co-habitants should be discussed. This is a time-consuming business but could be performed by a health visitor for the elderly. Therefore, whilst we advocate widespread vaccination, we acknowledge there are some situations where, with the patient's informed consent, it may be withheld.
1 Ashley J, Smith T, Dunnell K. Deaths in Great Britain associated with the influenza epidemic of Britain associated with the influenza epidem

2 Nguyen-van-Tam JS, Nicholson KG. Influenza Nguyen-van-Tam JS, Nicholson KG. Influenza
deaths in Leicestershire during the 1989-1990 deaths in Leicestershire during the $1989-1990$
epidemic: implications for prevention. Epidemiol Infect 1992; 108: 537-45.

3 Barker WH, Mulloolly JP. Influenza vaccination of elderly persons: reduction in pneumonia and influenza hospitalizations and deaths. $\mathscr{f} A M A$ 1980; 244: 2547-9.

4 Morgan R, King D, Turnbull CJ. Influenza vaccination: do the aged reap the benefit? Postgrad Med f 1995; 71: 122-3.

5 Nicholson KG. Immunisation against influenza among people aged over 65 living at home in Leicestershire during winter 1991-2. BMF 1993; 306: $974-6$.

6 Schoenbaum SC. A perspective of the benefits, costs and risks of immunisation. In: Weinstein costs and risks of immunisation. In: Weinstein L, Fields BN, eds. Seminars in infectious diseas

7 Helfrich BA, Segre M. Age related changes in the degeneracy of the mouse $T$ cell repertoire. Cell Immunol 1989; 118: 1 .

8 Depaoli P, Battistin S, Santini GF. Age related changes in human lymphocyte subsets: progressive reduction of CD4 CD45R population. Clin Immunol Immunopathol 1988; 48: 290.

9 Gillis S, Kozak R, Durante M. Immunological studies of agein. Decreased production of and response to $T$ cell growth factor by lymphocytes response to T cell growth factor by lymphocyte

10 Couch $R$. The effects of influenza on host defences. F Infect Dis 1981; 144: 284.

11 Brandstetter RD, Kazemi H. Ageing and the 1 Brandstetter RD, Kazemi H. Ageing and the
respiratory system. Med Clin North Am 1983; 67: respirat.

12 Puchelle E, Zahm JM, Bertrand A. Influence of age on bronchial mucocillary transport. Scand $\mathcal{F}$ Respir Dis 1979; 60: 307.
13 Raju L, Khan F. Pneumonia in the elderly. Geriatrics 1988; 43: 51 .

14 Comprehensive delivery of adult vaccination Minnesota, 1986-1992. MMWR 1993; 42: 768 70.

15 Strassburg MA, Greenland S, Sorvillo FJ, Lieb LE, Habel LA. Influenza in the elderly: report of an outbreak and a review of vaccine effectiveness reports. Vaccine 1986; 4: 38-44.

16 Govaert TME, This CTMCN, Sprenger MJW Dinant GJ, Knottnerus JA. The efficacy of influenza vaccinations in elderly individuals. randomized double blind placebo controlled trial. $¥ A M A$ 1994; 272: $1661-5$

17 Fedson DS, Wajada A, Nichol JP, Hammond GW, Kaiser DL, Roos LL. Clinical effectivenes of influenza vaccination in Manitoba. $¥ A M A$ 1993; 270: 1956-61.

18 Foster DA, Talsma A, Furumoto-Dawson A Influenza vaccine effectiveness in preventing Influenza vaccine effectiveness in preventing Am 7 Epidemiol 1992; 136: 296-307.

19 Mullooly J, Bennet M, Hornbrook M. Cost Mullooly J, Bennet $M$, Hornbrook $M$. Cost effectiveness of influenza vacination programs in an HMO: the experience of Kaiser Permante, Northwest Region. In: Hannoun C, Kendal AP,
Klenk HD, Ruben FL, eds. Options for control of influneza II. Amsterdam: Excerpta Medica, 1993; pp 53-62.

20 Nichol KL, Margolis KL, Wuorenma J, Von Sternberg $T$. The efficacy and cost effectivenes of vaccination against influenza among elderly persons living in the community. $N$ Eng 7 Med 1994; 331: 778-84.

21 Fedson D. Data watch. Health Affairs 1992; 3: 161-9.

22 Riddough MA, Sisk JE, Bell JC. Influenza vaccination: cost effectiveness and public policy. $¥ A M A$ 1983; 249: 3189 .
23 Arden NH, Patriarca PA, Kendal AP. Experiences in the use of inactivated influenza vaccine in nursing homes. In: Kendal AP, Patriarca PA eds. Options for the control of influenza. New York: Alan R Liss, 1986; p 155.

24 Beyer WEP, Palache AM, Baljet M. Antibody induction by influenza vaccines in the elderly: a induction by influenza vaccines in the elderly:

25 Gross PA, Quinnan GV, Weksler ME. Relation of chronic disease and immune response to of chronic disease and immune response to 303.

26 Powers PC, Sears SP, Murphy BR. Systemic and local antibody responses in elderly subjects given live or inactivated influenza $A$ virus vaccines. $f$ Clin Microbiol 1989; 27: 2666.

27 Govaert TM, Dinant GJ, Aretz K, Masurel N, Sprenger MJ, Knottnerus JA. Adverse reaction to influenza vaccination in elderly people: randomised double blind placebo controlled trial. BMF 1993; 307: 988-90.

28 Younkin S, Betts R, Roth F. Reduction in feve and symptoms in young adults with influenza $A$ and symptoms in young adults with influenza A aspirin or amantidine. Antimicrob Agents Chemother 1983; 23: 577 .

29 Dolin R, Reichmann R, Mandor H. A controlled trial of amantadine and rimantadine in the prophylaxis of influenza A infection. $N$ Engl $\mathcal{F}$ Med 1982; 307: 580.

30 Centres for Disease Control. Adult immunization: knowledge of attitudes, and practices. DeKalb and Foulton Counties, Georgia, 1988 MMWR 1988; 37: 657 . 